\title{
Multifunctional properties of monodisperse end-functionalized oligophenylenevinylenes*
}

\author{
M. S. Wong ${ }^{\ddagger}$ and Z. H. Li \\ Department of Chemistry, Hong Kong Baptist University, Kowloon Tong, \\ Hong Kong SAR, China
}

\begin{abstract}
Novel series of monodisperse, end-functionalized oligophenylenevinylenes (OPVs) containing up to eight phenyl rings have been designed and synthesized by a convergent approach using the stereoselective Wadsworth-Emmons reactions for an investigation of technologically useful functional properties. According to PM3 semiempirical quantum mechanical calculations, the frontier molecular orbitals and the energy gap of an OPV could be shifted or tuned by the functional substituent(s) that are incorporated as end-caps. Our theoretical and experimental evidences have shown that the end-substituents of an oligomer do not affect coplanarity of the $\pi$-conjugated system. It has also been found that the newly synthesized OPVs can exhibit various useful functional properties such as light-emitting properties, photovoltaic effects, third-order nonlinear optical responses, and chemical sensing properties depending on the nature of the end-substituents. In this contribution, the structural factor(s) that can enhance a specific functional property of a molecule or material will be discussed.
\end{abstract}

Poly(phenylenevinylene) (PPV) and its derivatives have been extensively explored and investigated for various technologically useful functional properties in the past two decades because of their potential applications in next-generation optoelectronic and photonic devices. Those properties include electroluminescent response [1], third-order optical nonlinearity [2], photovoltaic property [3], lasing effect [4], and chemical sensing [5]. PPV is the first $\pi$-conjugated semiconducting polymer that was used as an emissive material in organic light-emitting diodes (OLEDs) [6], and its derivatives can often exhibit an efficient electroluminescent response [7]. PPV thin-film with a long effective conjugated length was shown to have a large effective nonlinear refractive index, $\mathrm{n}_{2}$ and a desirable two-photon merit factor, $\mathrm{T}$ for all-optical switching and processing [8]. Upon mixing with electron-conducting buckminsterfullerene derivatives, a large photovoltaic response can be obtained [9]. Some PPV derivatives can even show good lasing and interesting chemical sensing properties. Nevertheless, it still remains a challenge for a chemist to further enhance and optimize a specific functional property of a PPV-based material for a practical application as subtle modification of the monomeric unit often leads to a dramatic change in optical and electronic properties of a polymer such as a fluorescence quantum yield and an energy gap. As a result, knowledge of the structure-functional property relationships is fundamentally important for a rational design of efficient and useful PPV-based functional materials. On the other hand, polymers always show structural inhomogeneities and imperfection regardless the method used to prepare them, which would hinder the structure-functional property investigations. Toward the goal of "functional property by design", there is an increasing interest in investigating structure-functional property rela-

\footnotetext{
*Lecture presented at the symposium "Polymers in electronics and photonics: Synthesis, characterizations and device applications", as part of the $39^{\text {th }}$ IUPAC Congress and $86^{\text {th }}$ Conference of the Canadian Society for Chemistry: Chemistry at the Interfaces, Ottawa, Canada, 10-15 August 2003. Other Congress presentations are published in this issue, pp. 1295-1603.

${ }^{\ddagger}$ Corresponding author
} 
tionships of monodisperse, well-defined oligomers (i.e., OPVs) recently as they can serve as model compounds to the related polymers [10,11]. In addition, functionalized oligomers can be easily obtained in high purity and fabricated into a thin film, which provides advantages of using them as an active material for various device applications such as OLEDs, plastic solar cell, and field-effect transistors [12-14].

Because of the rigidity and planarity of the repeating phenylenevinylene units, unsubstituted PPV and the higher homologs of OPVs are highly insoluble. One widely employed approach to enhance the solubility and processability of an oligomer or a polymer is to incorporate lateral substitutent(s) such as alkyl or alkoxy groups onto the $\pi$-conjugated main chain. However, the introduction of lateral substituent(s) often causes a distortion of the coplanarity of the $\pi$-conjugated backbone due to steric hindrance and hence affecting favorable physical properties of an oligomer such as the fluorescence quantum yield, the nonlinear refractive index, and the turn-on voltage in OLEDs. For instance, Hadziioannou et al. have synthesized several 5-phenyl-ring OPV derivatives bearing various side-groups including cyano, octyl, or octyloxy on the central part of the main [15]. The X-ray structural analyses have shown that the central phenyl ring of these OPV derivatives is greatly twisted off from coplanarity with the adjacent phenyl rings [16]. They have also found that the relative position of the side-substituents has a profound effect on the luminescent properties and the single-layer OLED based on unsubstituted 5-phenyl-ring oligomer shows superior device performance compared to that of the octyloxy-side-substituted counterparts [17]. Meier et al. have synthesized a series of soluble 2,5-dipropoxy-substituted OPVs containing up to 12-phenyl rings using a combination of the Wittig-Horner, Siegrist, and McMurry reactions [18]. The absorption maxima of these OPVs become saturated when the oligomer contains more than 12-phenyl rings and are greatly red-shifted compared to those of the corresponding unsubstituted and end-substituted ones. Single-layer OLEDs fabricated by these OPVs blended in a polystryene matrix exhibit a progressive decrease in current onset with an increase in chain length [19]. Yu et al. have used an orthogonal approach to stepwise synthesize a series of OPVs bearing solubilizing 2,5-dioctyloxy substituents on the alternative phenyl rings by means of Heck and Horner-Wadsworth-Emmons reactions for fluorescent and mesogenic investigations. The saturation of<smiles>[R]c1cc(/C=C/c2ccc(/C(C)=C/c3cc([R])c([R])c([R])c3)cc2)cc([R])c1[R]</smiles>

OPV(n)-OR

$\mathrm{n}=1-4: \mathrm{R}_{1}=\mathrm{CH}_{3} \mathrm{O}, \mathrm{R}_{2}=\mathrm{C}_{4} \mathrm{H}_{9} \mathrm{O}\left(\mathrm{CH}_{2} \mathrm{CH}_{2} \mathrm{O}\right)_{2}$ $\mathrm{n}=5-6: \mathrm{R}_{1}=\mathrm{R}_{2}=\mathrm{C}_{4} \mathrm{H}_{9} \mathrm{O}\left(\mathrm{CH}_{2} \mathrm{CH}_{2} \mathrm{O}\right)_{2}$

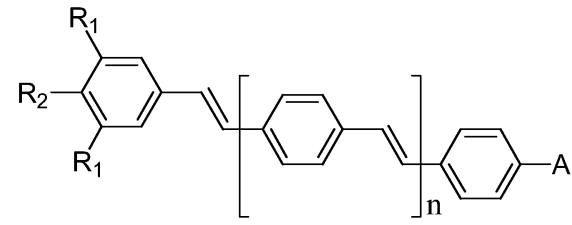

OPV(n)-ORSO

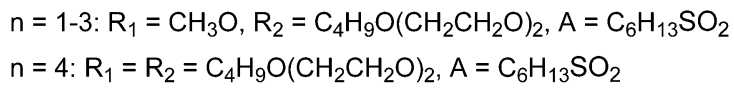

OPV(n)-ORNO

$$
\begin{aligned}
& n=0-1: R_{1}=H, R_{2}=C_{4} H_{9} O\left(C_{2} C_{2} O\right)_{2}, A=N_{2} \\
& n=2: R_{1}=C_{3} O, R_{2}=C_{4} H_{9} O\left(C_{2} C_{2} O\right)_{2}, A=N_{2}
\end{aligned}
$$


the absorption maximum occurs after the conjugation length reaches 8-phenyl rings in this series [20]. Very recently, Luo et al. have synthesized the longest laterally substituted OPV containing 17-phenyl rings with 2,5-dihexyloxy groups on the alternative phenyl rings and two methyl sulfide end groups using iterative coherent approach [21]. As a result, it would be of great interest to conserve the integrity of the coplanarity of the phenylenevinylene $\pi$-conjugated backbone of OPVs in order to preserve their unique characteristics and desirable functional properties. To enhance the solubility and avoid disrupting the coplanarity of $\pi$-conjugated framework, end-substitution approach could be used. Müllen and coworkers have synthesized a series of soluble OPVs end-substituted with tert-butyl groups at the nonconjugated positions of the main chain for the charge-storage mechanism and related spectroscopic studies $[22,23]$. They have also found that the absorption maximum of the 6-phenyl-ring oligomer begins to converge [24].

In the last few years, we have designed and synthesized series of soluble and highly coplanar OPVs either symmetrically or asymmetrically end-capped with various functional substituents such as polyalkyleneoxy, alkylsulfanyl, alkylsulfonyl, or nitro groups. In addition, the influence of substitution and the conjugation length effect on various physical properties were theoretically and experimentally investigated. It is important to find that depending on the electronic property of substituents, the endfunctionalized OPVs can exhibit various useful functional properties, which include third-order nonlinear optical responses [25,26], light-emitting properties [27-29], photovoltaic effects [30], and chemical sensing properties [31]. In this contribution, an overview of our ongoing effort on probing the structural factor(s) that can enhance a specific functional property of end-functionalized OPVs is discussed.

With the use of multiple solubilizing polyalkyleneoxy substituents, highly soluble OPVs containing up to 8-phenyl rings have been prepared. According to the PM3 semiempirical calculations, the phenylenevinylene units in the optimized geometry of the 7-phenyl-ring OPV bearing hexamethoxy groups at the ends are highly coplanar. Figure 1 shows the top and side views of the PM3-optimized geometry of the 7-phenyl-ring OPV. In general, the convergent synthetic strategy was employed to construct the OPV skeleton, and the stereoselective Wadsworth-Emmons reaction was used as a key step to synthesize the all-trans carbon-carbon double bonds of an oligomer. In the symmetrically disubstituted OPV series, the odd-phenyl-ring oligomers were synthesized by a convergent $[\mathrm{N}+1+\mathrm{N}]$ approach, the double Wadsworth-Emmons reactions of bis(diethyl) $p$-xylylene[bis-(phosphonate)] and the corresponding aldehydes [i.e., $3+1+3$ for 7-phenyl-ring oligomer, OPV(5)-OR]. And the evenphenyl-ring oligomers were prepared by a convergent $\left[\mathrm{N}+\mathrm{N}^{\prime}\right]$ approach [i.e., $4+4^{\prime}$ for 8-phenyl-ring oligomer, OPV(6)-OR]. Similarly, the entire homolog series of asymmetrically disubstituted OPVs [e.g., OPV $(n)$-ORSO, $n=1-4$ ] was synthesized by a $[\mathrm{M}+\mathrm{N}]$ approach as summarized in Scheme 1.

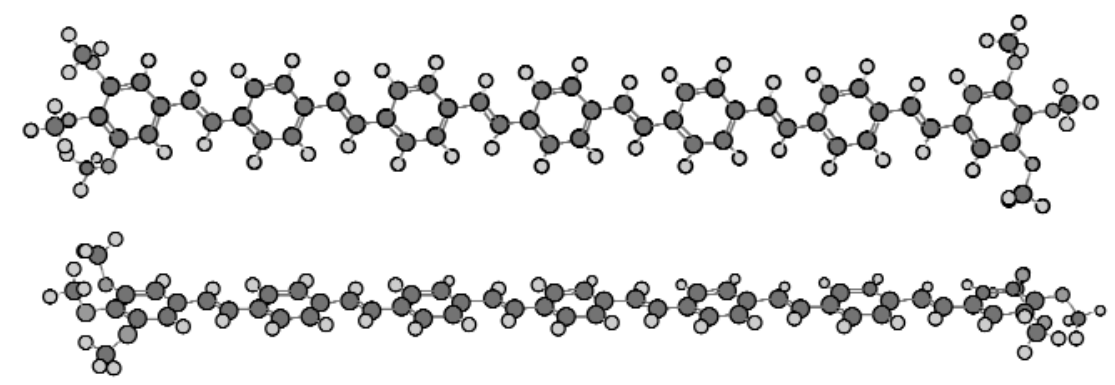

Fig. 1 Top and side views of the PM3-optimized geometry of the 7-phenyl-ring OPV. 
$[\mathrm{N}+1+\mathrm{N}]$ Approach:

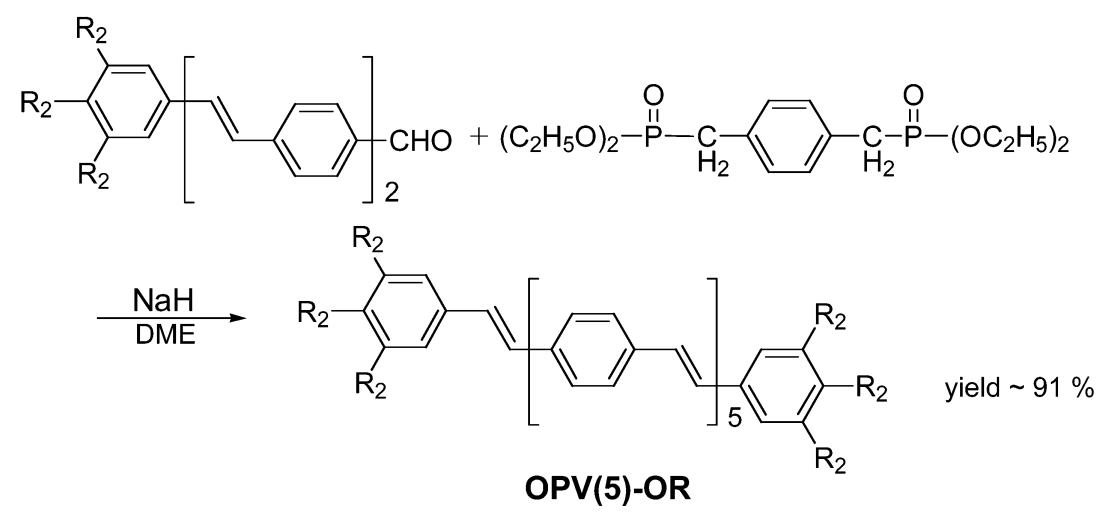

$\left[\mathrm{N}+\mathrm{N}^{\prime}\right]$ Approach:
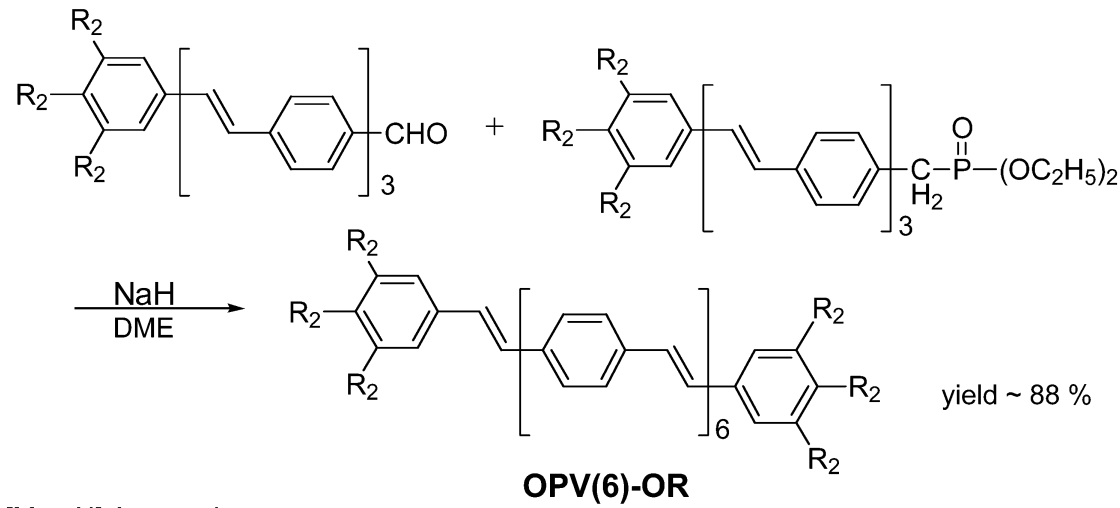

$[M+N]$ Approach:
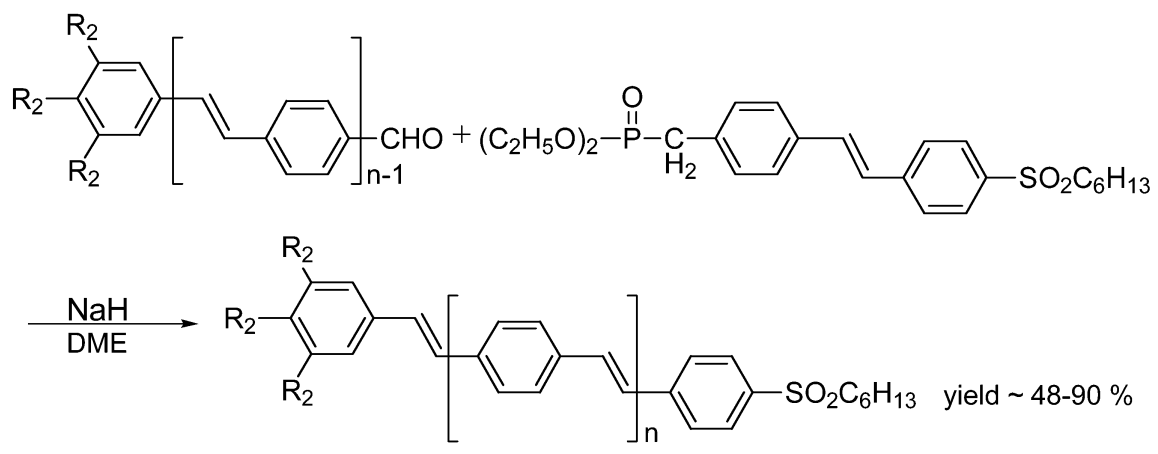

$\mathrm{R}_{2}=\left(\mathrm{OCH}_{2} \mathrm{CH}_{2}\right)_{2} \mathrm{OC}_{4} \mathrm{H}_{9} \quad \mathrm{OPV}(\mathrm{n})$-ORSO $\quad \mathrm{n}=1-4$

Scheme 1 Synthetic strategy for the preparation of symmetrically and asymmetrically end-functionalized OPVs. 
It was found from the PM3 semiempirical calculations that the energy gap of both methoxy-disubstituted and unsubstituted 3-phenyl-ring OPVs show a tendency of saturation as the chain length increases indicating the existence of an effective conjugated length for the energy gap (Fig. 2). On the other hand, the methoxy donating groups reduce the energy gaps of 4,4'-dimethoxy-substituted OPVs approaching a limit of convergence faster than the unsubstituted counterparts. This implies that the methoxy-disubstituted OPV series may have a shorter effective conjugated length for the energy gap. Consistent with the predicted trend, the alkoxy donating groups end-capped at the conjugated positions of OPVs promote a faster approach of convergence in absorption maxima (or energy gap). Furthermore, the absorption maxima of the asymmetrically disubstituted OPVs bearing alkoxy group at one end and alkylsulfonyl group at the other end converge even faster than the alkyoxy-disubstituted one (Fig. 2).

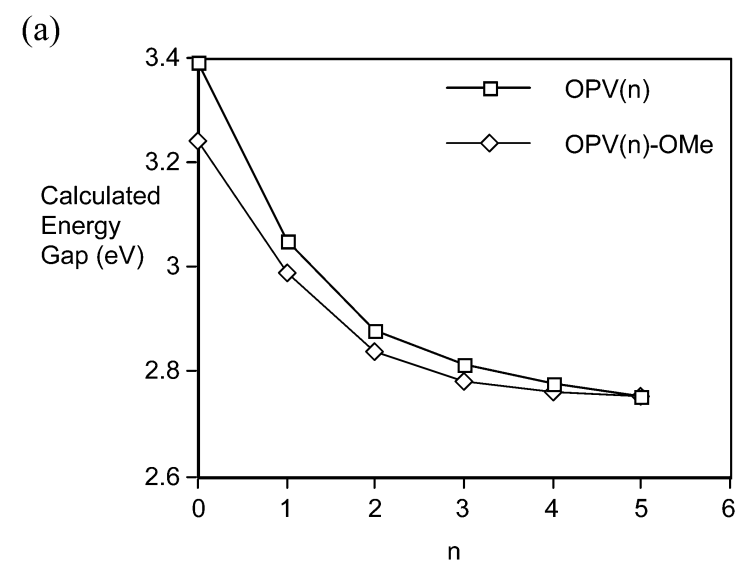

(b)

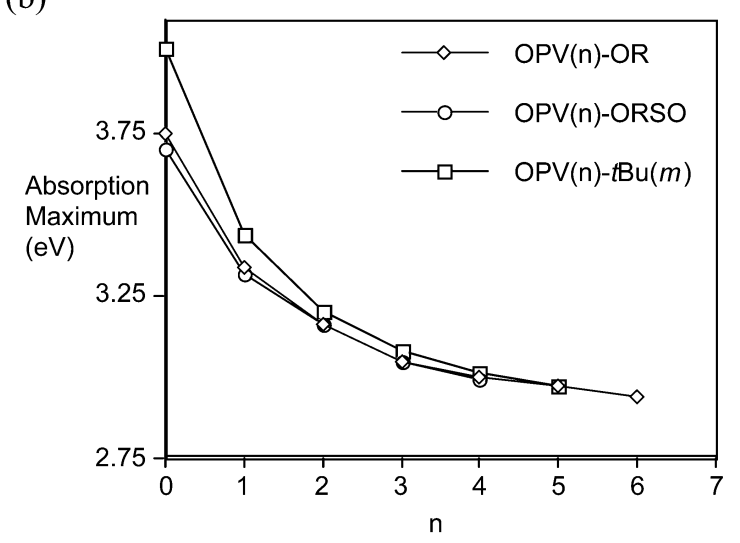

Fig. 2 (a) Plots of PM3 calculated energy gap of unsubstituted OPVs [OPV $(n)]$ and 4,4'-dimethoxy-substituted OPVs [OPV $(n)$-Ome] against number of phenylene-vinylene units, $n$. (b) Plots of absorption maxima of $\mathrm{OPV}(n)-\mathrm{OR}, \mathrm{OPV}(n)-\mathrm{ORSO}$ and $\mathrm{OPV}(n)-t-\mathrm{Bu}(m)$ (from ref. [22]) against number of phenylenevinylene units, $n$.

According to our PM3 semiempirical calculations, the frontier molecular orbitals of an oligomer (i.e., a 3-phenyl-ring OPV) can be shifted by the functional substituents incorporated at the ends of the $\pi$-conjugated skeleton as shown in Fig. 3. In general, electron-donating groups attached at the conjugated positions destabilize the frontier molecular orbitals, shifting the highest occupied molecular orbital (HOMO) and the lowest unoccupied molecular orbital (LUMO) levels upward relative to the unsubstituted one. While the incorporation of the electron-withdrawing groups stabilizes the HOMO and 


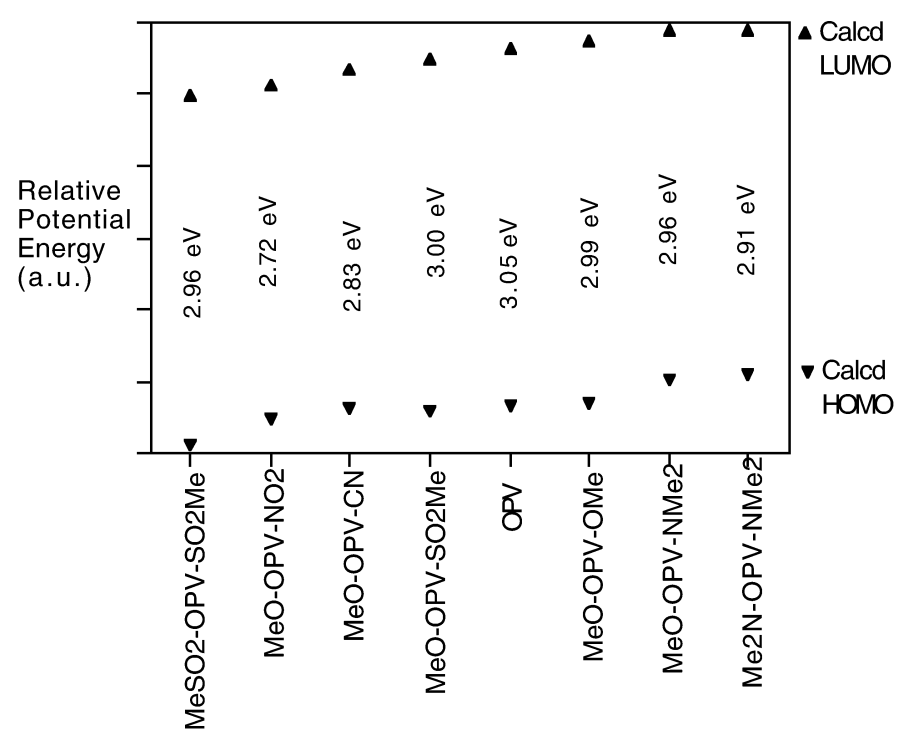

Fig. 3 Plots of HOMO and LUMO energy levels calculated by PM3 semiempirical quantum mechanical calculations against 4,4'-disubstituted 3-phenyl-ring OPVs. The energy gaps shown were corrected by solvent interaction (from ref. [1]).

LUMO levels, shifting the levels downward relative to the unsubstituted one (Fig. 3). However, the stabilization (or destabilization) of the HOMO and the LUMO levels are in different extent. This leads to an overall decrease in the energy gap of an end-functionalized oligomer when compared to that of an unsubstituted one. These results imply that the HOMO and LUMO levels as well as the energy gap of an oligomer can be tuned by the end-substituents.

The molecular nonlinearity $\left(\gamma_{\text {calc }}\right)$ predicted by the finite field method in MOPAC6 and the timedependent coupled perturbed Hartree-Fock method within the MOPAC93 package showed that $\gamma_{\text {calc }}$ increases with an extension of chain length, but shows no sign of saturation in contrast to the energy gap (Fig. 4). In addition, the $\gamma$ enhancement contributed by the end-substituents becomes comparatively less significant as the chain length increases. On the other hand, the $\gamma_{\text {calc }}$ differences between the symmetrically and asymmetrically disubstituted OPVs are relatively small. We have also determined the absolute $|\gamma|$ by open and closed aperture Z-scan technique using $800 \mathrm{~nm}$ fs laser pulses in two different solvents, THF, and chloroform for both OPV $(n)$-OR and OPV (n)-ORSO series. Consistently, the Z-scan determined $\gamma$ enhances with an increase in the conjugation length in both series, reaffirming an importance of the extended delocalization to enhance $\gamma$, but there is a sign of saturation of $\gamma$ in the asymmetrically disubstituted OPV series. The $\gamma$ enhancement due to the asymmetric substitution, namely, the cascade effect, has found to be significant for the lower homologs of OPVs. A very large $|\gamma|$ value, up to $2.5 \times 10^{-32} \mathrm{esu}$, was obtained in the symmetrically disubstituted OPVs, suggesting the importance of the coplanarity and long conjugated length.

It is worth mentioning that most of the newly synthesized end-functionalized OPVs are highly luminescent which is presumably due to the rigidity and coplanarity of the phenylenevinylene units. Multilayer OLEDs with structure of (ITO/TPD/OPV/PBD or LiF/Al) where TPD is $N, N^{\prime}$-diphenyl$N, N^{\prime}$-bis(3-methylphenyl)-1,1-biphenyl-4,4'-diamine and PBD is 2-(4-biphenyl)-5-(4-tertbutylphenyl)-1,3,4-oxadiazole using end-functionalized 3-phenyl-ring OPV as a light-emitting layer were fabricated and investigated. In addition to the emission color, the OLED stability and performance are significantly influenced by the end-substituents. It is important to note that OPV(1)-SO-based OLED device was found to be less stable. For OPV(1)-OR-based double-layer devices which emit with a peak at $488 \mathrm{~nm}$, the electroluminescence (EL) efficiency is about $0.2 \mathrm{~cd} / \mathrm{A}$. With an insertion of LiF 

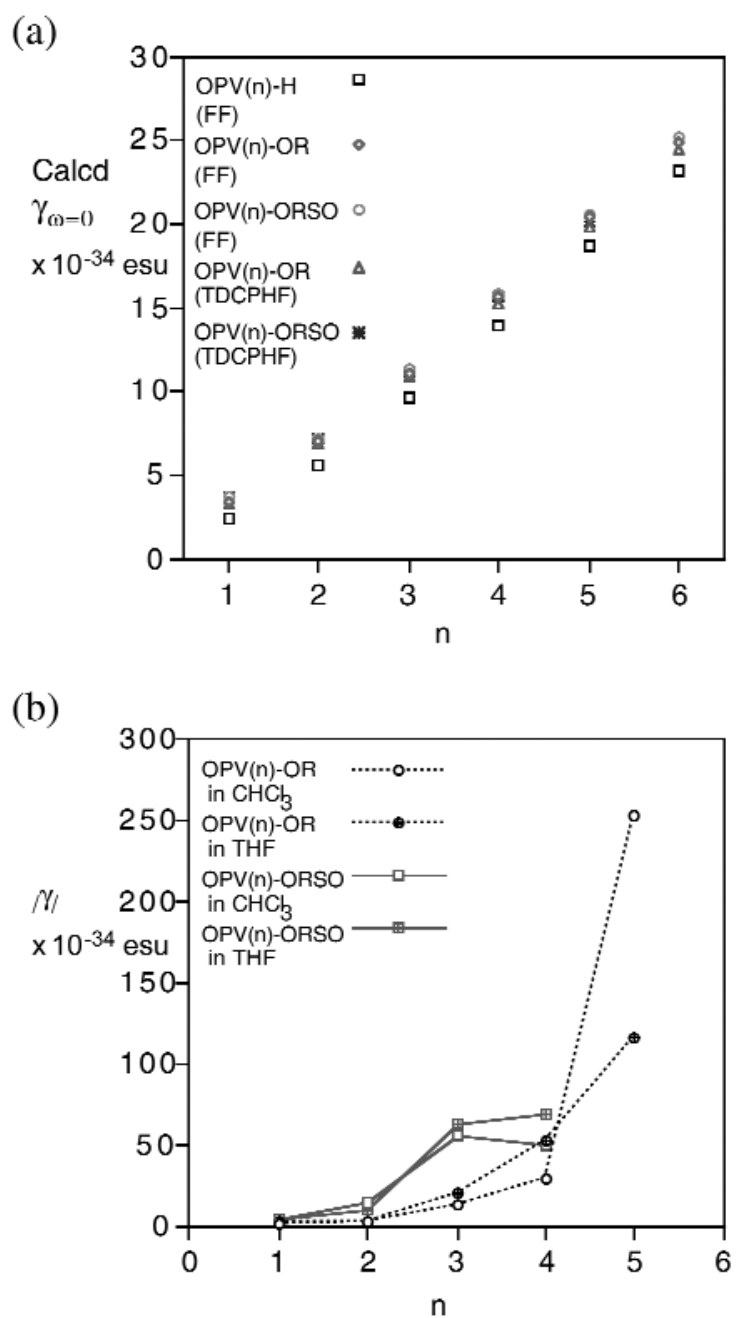

Fig. 4 (a) Plots of $\gamma_{\omega=0}$ calculated by the finite field method (FF) and the time-dependent coupled perturbed Hartee-Fock method (TDCPHF) of OPV $(n)-\mathrm{H}$, OPV $(n)-\mathrm{OR}$, and OPV $(n)$-ORSO against the phenylenevinylene unit, $n$. (b) Plots of Z-scan determined $|\gamma|$ in THF and chloroform against the phenylenevinylene unit, $n$.

as an electron injection-enhancing layer, the efficiency increases to $0.47 \mathrm{~cd} / \mathrm{A}$. Upon replacement of $\mathrm{LiF}$ layer with PBD as a hole-blocking layer, the efficiency further improves to $1.9 \mathrm{~cd} / \mathrm{A}$ with a brightness of $700 \mathrm{~cd} / \mathrm{m}^{2}$. On the other hand, OPV(1)-ORSO-based devices emit at $519 \mathrm{~nm}$ (Fig. 5). In a doublelayer device, the EL efficiency reaches $0.7 \mathrm{~cd} / \mathrm{A}$. With an addition of a LiF layer, the efficiency increases up to $2.3 \mathrm{c} / \mathrm{A}$ with a brightness of $600 \mathrm{~cd} / \mathrm{m}^{2}$. The superior performance of OPV(1)-ORSO-based device was attributed to the electron-withdrawing nature of alkylsulfonyl functionality, which lowers the LUMO level of the emitting layer leading to a better balance of charge injection/transport of the OPV(1)-ORSO-based device. These findings suggest that donor-acceptor type luminescence materials may render to improve the intrinsic imbalanced charge character of the OLEDs.

Furthermore, the highly fluorescent end-functionalized OPVs may be useful for fluorescent sensing if end-substituents can act as receptors to recognize target molecules. And upon binding of fluorophores with target molecules, there is a change in the spectral property (i.e., fluorescent quenching of the fluorophores), which can gives rise to a sensing mechanism. We have found that hexaalkylsulfanyl substituents incorporated at the ends of OPV skeleton can act as receptors or ionophores to selectively 
(a)

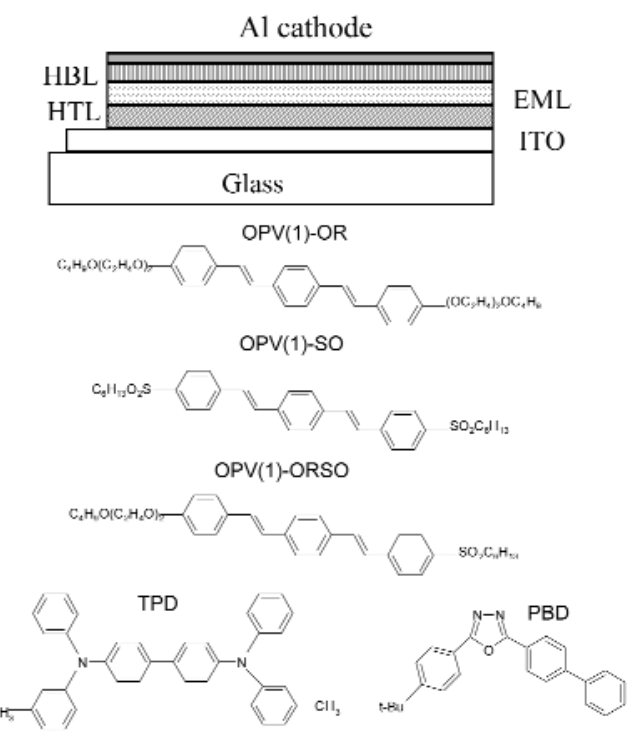

(b)

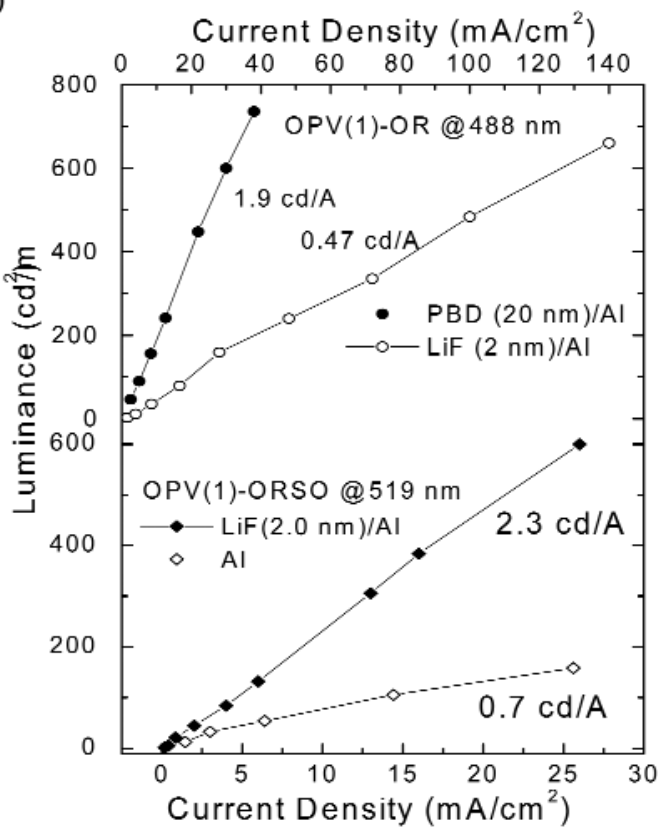

Fig. 5 (a) A typical OLED structure fabricated and the molecular structures involved in this study. (b) EL efficiency of OPV(1)-OR-based OLEDs and OPV(1)-ORSO-based OLEDs with structure of ITO/TPD/LiF or PDB/Al.

recognize silver(I) cation in membrane optode. Upon contacting with silver(I) ion solution, there is a strong fluorescent quenching in the OPV(1)-SR-based optode sensor. Importantly, the fluorescence intensity decreases with an increase in silver(I) ion concentration and there is no such response toward other heavy metal ions such as $\mathrm{Zn}^{2+}, \mathrm{Cd}^{2+}, \mathrm{Cu}^{2+}, \mathrm{Co}^{2+}$, and $\mathrm{Pb}^{2+}$. More interestingly, the longer oligomer OPV(2)-SR-based sensor also shows the same selectivity toward silver(I) ion with more than two-fold enhanced sensitivity (Fig. 6). The superior sensitivity of OPV(2)-SR-based sensor is attributed 
(a)

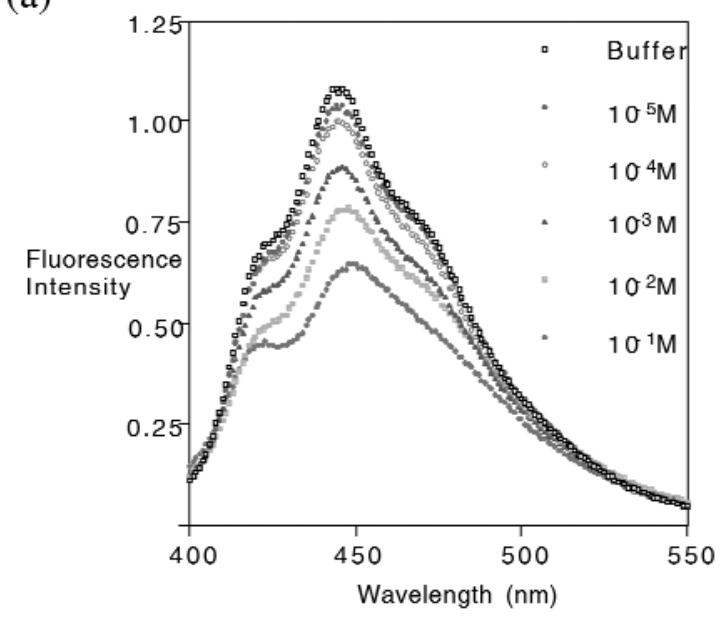

(b)

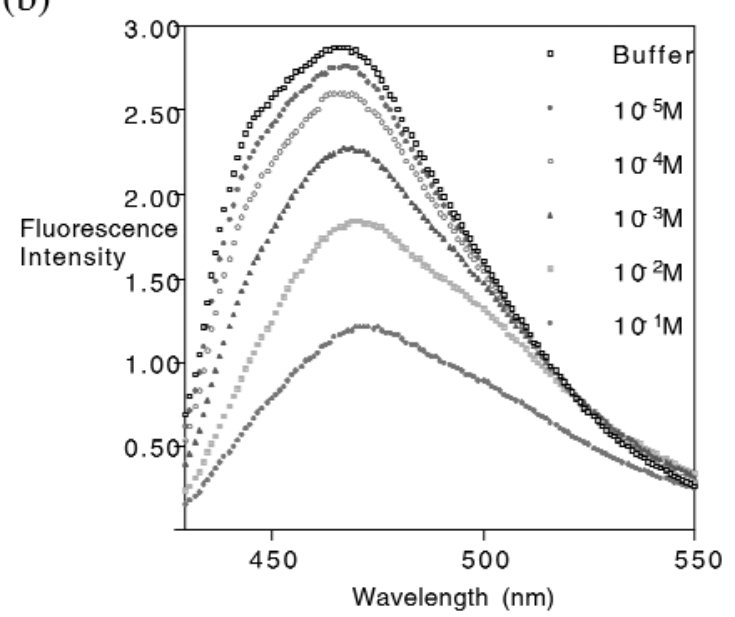

Fig. 6 (a) Fluorescence response of OPV(1)-SR-based membrane optode sensor toward various concentration of $\mathrm{Ag}^{+}$ion. (b) Fluorescence response of OPV(2)-SR-based membrane optode sensor toward various concentration of $\mathrm{Ag}^{+}$ion.

to the substantially higher fluorescence quantum yield of OPV(2)-SR oligomer $(\Phi=0.65)$ than that of OPV(1)-SR ( $\Phi=0.15)$.

In contrast to most of end-functionalized OPVs we synthesized so far, OPVs bearing polyalkyleneoxy at one end and nitro group at the other end exhibit very low fluorescence $(\Phi=0.01-0.04)$. Multilayer OLEDs based on this OPV series also exhibit very low light emission and short device lifetime. However, such devices show a clear rise in reverse current upon an increase in illumination as shown in Fig. 7, suggesting the photovoltaic phenomenon, which was not observed in other donor-acceptor OPV series. The spectral response of such an effect correlates well with the absorption characteristics of OPV(n)-ORNO oligomer in which the photocurrent reaches a maximum at the absorption maximum of an oligomer. Furthermore, the photovoltaic responsivity enhances with an increase in conjugation length. Replacing the OPV backbone with the oligophenylene $\pi$-conjugated core (i.e., terphenyl), the photocurrent response of such a photovoltaic cell is substantially reduced, signifying the importance of highly coplanar $\pi$-conjugated system. These results also imply that the spectral response and the photovoltaic sensitivity can be modified or tuned by the type and length of the $\pi$-conjugated 
(a)

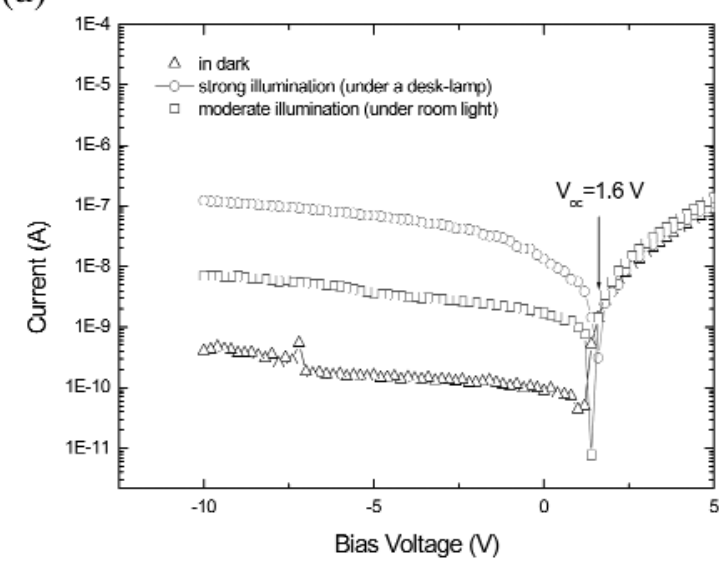

(b)

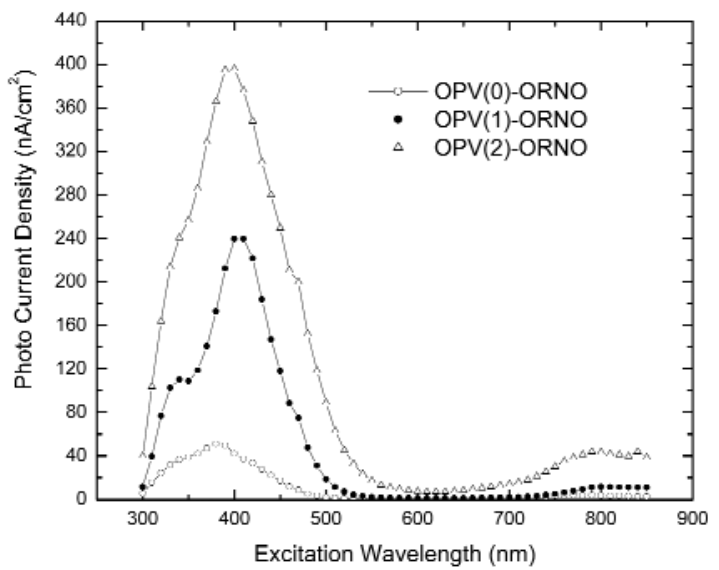

Fig. 7 (a) Current-voltage characteristics of OPV(1)-ORNO-based photovoltaic cell. (b) Photocurrent densityexcitation wavelength characteristics of the $\mathbf{O P V}(\boldsymbol{n})$-ORNO-based photovoltaic devices.

core. The mechanism of this effect is not fully known yet. Presumably, the strong electronic-withdrawing property of the nitro group coupled with the polyalkyleneoxy electron donor enhances and stabilizes the charge separation leading to fluorescence quenching. The dissociation and migration of charges to the opposite electrodes give rise to the photocurrent.

In summary, we have demonstrated that the functional properties of oligophenylene-vinylene can be easily tuned by functional substituents incorporated at the ends. In addition, OPVs end-functionalized with proper substituents can be useful for third-order optically nonlinear, light-emitting, photovoltaic, and chemical sensing applications.

\section{ACKNOWLEDGMENT}

This work was partially supported by an Earmarked Research Grant (HKBU2051/01P) from the Research Grants Council, Hong Kong. 


\section{REFERENCES}

1. A. Kraft, A. C. Grimsadle, A. B. Holmes. Angew. Chem., Int. Ed. 37, 402-428 (1998).

2. A. Samoc, M. Samoc, M. Woodruff, B. Luther-Davies. In Photonic Polymer Systems, D. L. Wise, G. Wnek, D. J. Trantolo, T. M. Cooper, D. J. Gresser (Eds.), pp. 373-476, Marcel Dekker, New York (1998).

3. C. J. Brabec, N. S. Sariciftci, J. C. Hummelen. Adv. Funct. Mater. 11, 15-26 (2001).

4. F. Hide, M. A. Diaz-Garcia, B. J. Schwartz, A. I. Heeger. Acc. Chem. Res. 30, 430-436 (1997).

5. M. Kimura, T. Horai, K. Hanabusa, H. Shirai. Adv. Mater. 10, 459-462 (1998).

6. J. Burroughs, D. D. Bradley, A. R. Brown, R. N. Marks, K. Mackey, R. H. Friend, P. L. Burns, A. B. Holmes. Nature 347, 539-541 (1990).

7. R. H. Friend, R. W. Gymer, A. B. Holmes, J. H. Burroughes, R. N. Marks, C. Taliani, D. D. C. Bradley, D. A. Dos Santos, J. L. Brédas, M. Lögdlund, W. R. Salaneck. Nature 397, 121-128 (1999).

8. A. Samoc, M. Samoc, M. Woodruff, B. Luther-Davies. Opt. Lett. 20, 1241-1243 (1995).

9. S. E. Shaheen, C. J. Brabec, N. S. Sariciftci, F. Padinger, T. Fromherz, J. C. Hummelen. Appl. Phys. Lett. 78, 841-843 (2001).

10. K. Müllen and G. Wegner. Electronic Materials: The Oligomer Approach, Wiley-VCH, Weinheim (1998).

11. R. E. Martin and F. Diederich. Angew. Chem., Int. Ed. 38, 1350-1377 (1999).

12. Y. Shirota. J. Mater. Chem. 10, 1-25 (2000).

13. U. Mitschke and P. Bäuerle. J. Mater. Chem.10, 1471-1507 (2000).

14. J. L. Segura and N. Martin. J. Mater. Chem. 10, 2403-2453 (2000).

15. R. E. Gill, A. Meetsma, P. F. van Hutten, G. Hadziioannou. Chem. Mater. 8, 1341-1346 (1996).

16. R. E. Gill, A. Meetsma, G. Hadziioannou. Adv. Mater. 8, 212-214 (1996).

17. P. F. van Hutten, J. Wildeman, A. Meetsma, G. Hadziioannou. J. Am. Chem. Soc. 121, 5910-5918 (1999).

18. U. Stalmach, H. Kolshom, I. Brehm, H. Meier. Liebigs Ann. 1449-1456 (1996).

19. V. Gebhardt, A. Bacher, M. Thelakkat, U. Stalmach, H. Meier, H. W. Schmidt, D. Haarer. Adv. Mater. 11, 119-123 (1999).

20. T. Maddux, W. Li, L. Yu. J. Am. Chem. Soc. 119, 844-845 (1997).

21. C. Xue and F.-T. Luo. J. Org. Chem. 68, 4417-4421 (2003).

22. J. Heinze, J. Mortensen, K. Müllen, R. Schenk. J. Chem. Soc. Chem. Commun. 701-703 (1987).

23. R. Schenk, G. H., K. Meerholz, J. Heinze, K. Müllen. J. Am. Chem. Soc. 113, 2634-2647 (1991).

24. U. Scherf and K. Müllen. Synthesis 23-38 (1992).

25. M. S. Wong, M. Samoc, A. Samoc, B. Luther-Davies, M. G. Humphrey. J. Mater. Chem. 8, 2005-2009 (1998).

26. M. S. Wong, Z. H. Li, M. F. Shek, M. Samoc, A. Samoc, B. Luther-Davies. Chem. Mater. 14, 2999-3004 (2002).

27. Y. Tao, A. Donat-Bouillud, M. D’Iorio, L. J. Lam, T. C. Gorjanc, C. Py, M. S. Wong. Synth. Met. 111-112, 417-200 (2000).

28. Y. Tao, A. Donat-Bouillud, M. D’Iorio, J. Lam, T. C. Gorjanc, C. Py, M. S. Wong, Z. H. Li. Thin Solid Films 363, 298-301 (2000).

29. M. S. Wong, Z. H. Li, M. F. Shek, K. H. Chow, Y. Tao, M. D’Iorio. J. Mater. Chem. 10, 1805-1810 (2000).

30. M. S. Wong, Z. H. Li, Y. Tao, M. D'Iorio. Chem. Mater. 15, 1198-1203 (2003).

31. M. S. Wong, W. H. Chan, W. Y. Chan, J. Li, X. Dan. Tetrahedron Lett. 41, 9293-9297 (2000).

32. U. Mitschke, E. M. Osteritz, T. Debaerdemaeher, M. Sokolowski, P. Bäuerle. Chem. Eur. J. 4, 2211-2224 (1998). 